\title{
Component Load
}

National Cancer Institute

\section{Source}

National Cancer Institute. Component Load. NCI Thesaurus. Code C49340.

The correlation coefficients between variable and factors. 